\title{
Performance Evaluation of an IEEE 802.15.4a Physical Layer with Energy Detection and Multi-User Interference
}

\author{
Manuel Flury, Ruben Merz, Jean-Yves Le Boudec and Julien Zory ${ }^{\dagger}$ \\ EPFL, School of Computer and Communication Sciences \\ $\dagger$ STMicroelectronics \\ \{manuel.flury, ruben.merz, jean-yves.leboudec\}@epfl.ch julien.zory@st.com
}

\begin{abstract}
-
We evaluate the performance of an IEEE 802.15.4a ultra-wide band (UWB) physical layer, with an energy-detection receiver, in the presence of multi-user interference (MUI). A complete packet based system is considered. We take into account packet detection and timing acquisition, the estimation of the power delay profile of the channel, and the recovery of the encoded payload. Energy detectors are known to have a low implementation complexity and to allow for avoiding the complex channel estimation needed by a Rake receiver. However, our results show that MUI severely degrades the performance of the energy detection receiver, even at low traffic rate. We demonstrate that using an IEEE 802.15.4a compliant energy detection receiver significantly diminishes one of the most appealing benefits of UWB, namely its robustness to MUI and thus the possibility to allow parallel transmissions. We further find that timing acquisition and data decoding both equally suffer from MUI.
\end{abstract}

\section{INTRODUCTION}

The IEEE 802.15.4 standard targets low data rate wireless networks with extensive battery life and very low complexity. Its current physical layer is based on a narrowband radio, operating in the unlicensed ISM band at $2.4 \mathrm{GHz}$. The IEEE 802.15.4a [1] proposal is an amendment to the 802.15.4 specification. It will add an impulse-radio ultra-wide band (IRUWB) physical layer [2] that could operate in several bands of $500 \mathrm{MHz}$ (or $1.5 \mathrm{GHz}$ ) from approximately $3 \mathrm{GHz}$ to $10 \mathrm{GHz}$. This physical layer should offer a better robustness against interference and multipath propagation channels, a higher datarate, and the possibility to perform ranging between devices. The IEEE 802.15.4a proposal allows for implementing either, a coherent receiver (for instance, a Rake receiver), or a noncoherent receiver (for instance, based on energy-detection).

Due to its relative low-complexity [3] an energy detection receiver is of interest for sensor network applications where devices should be inexpensive and have extremely low power consumption. For a coherent Rake receiver a high sampling frequency is required in order to properly estimate the channel characteristics [4]. On the contrary, an energy detection receiver might only need to estimate the channel power delay profile, can generally operate at a lower sampling frequency

The work presented in this paper was supported (in part) by the National Competence Center in Research on Mobile Information and Communication Systems (NCCR-MICS), a center supported by the Swiss National Science Foundation under grant number 5005-67322, and by CTI contract No7109.2;1 ESPP-ES and is robust against timing impairments [3]. Unfortunately, it is less robust to interference than a coherent receiver. Indeed, it cannot take advantage of the full diversity offered by the ultra-wide band channel.

Our objective in this paper is to evaluate the performance of a complete IEEE 802.15.4a IR-UWB physical layer implementation with an energy-detector receiver and multi-user interference (MUI).

MUI occurs due to concurrent packet transmissions. The mandatory medium access control (MAC) protocol in the IEEE 801.15.4a amendment is Aloha (without any form of clear channel assessment). In such a case, concurrent transmissions inevitably occur. The choice of a simple Aloha protocol is justified by the potential robustness of UWB to interference and by the infrequent nature of transmissions. In this paper we show that the former is not necessarily true for an energy detection receiver. We find that an 802.15.4a compliant energy detection receiver only shows a very limited capture effect. Its performance is quite close to a case where packets are lost if several transmissions are active concurrently. In a near-far scenario with one strong interferer we even have no capture effect at all.

Due to the complexity of the scenarios that we study, we do the performance evaluation through extensive simulations. The code used for our simulations is readily available [5].

The remainder of this paper is organized as follows; in Section II, we describe the receiver architecture that we consider and give the necessary details about the IEEE 802.15.4a physical layer to understand the results of the performance evaluation. In Section III, we first introduce the scenarios and parameters of the simulations. Then, we present the results of our performance evaluation. Related work is presented in Section IV. Finally, we conclude this paper in Section V.

\section{SYSTEM MODEL}

In this section, we describe the architecture of the receiver that we consider. We also give the necessary details about the IEEE 802.15.4a standard to understand the performance evaluation in Section III.

As in a classical IR-UWB physical layer [2], the IEEE 802.15.4a proposal uses time-hopping to smooth the spectrum of the signal and to mitigate the possible impact of multiuser interference. With an energy-detection receiver, compliant 
devices should use binary pulse position modulation (BPPM). The main difference with respect to a classical IR-UWB physical layer lies in the signal format of the data frames. Instead of sending a single pulse per frame, a short, continuous burst of pulses with pseudo-random polarity is sent.

An IEEE 802.15.4a packet consists of two parts: the preamble part and the payload. For a payload of $L_{p}$ bits, the transmitted signal is made of $L_{p}$ frames. A frame is further divided into $N_{c}$ chips of $T_{c}$ seconds. In each frame, a single burst of $N_{c p b}$ pulses $\left(N_{c b p}=4\right.$ for the mandatory mode we consider in this paper) is transmitted. A burst of pulses is the concatenation of $N_{c p b}$ pulses, whose amplitudes are modulated by a binary scrambling sequence. The location of the burst inside the $l$ th frame depends on the $l$ th data bit $d_{l}$ and on the time-hopping sequence; it is a sequence $\left[c_{o}, c_{1}, \ldots, c_{L_{p}-1}\right]$ of integers chosen in $\left\{0,1, \ldots, N_{h o p}-1\right\}$ where $N_{h o p}=\frac{N_{c}}{4 N_{c p b}}$. The burst inside the $l$ th frame is timeshifted by $\left(c_{l} \cdot N_{c p b}+d_{l} \cdot N_{c} / 2\right) T_{c}$ seconds with respect to the beginning of the frame. The scrambling sequences and the time-hopping sequences are generated by a linear feedback shift register (LFSR) as described in the 802.15.4a proposal [1]. Note that the LFSR is initialized to the same state for the transmission of each packet. Hence, all transmitters have the same scrambling and time-hopping sequence.

In this article, we focus on the mandatory data rate of 0.85 $\mathrm{Mbit} / \mathrm{s}$, with the low pulse-repetition frequency (LPRF) mode. The values of $N_{c}, T_{c}, N_{c p b}$, and $N_{h o p}$ for the LPRF mode are given in Table II. A $(55,63)$ Reed-Solomon (RS) code is used for error-correction [1].

A preamble is added before the payload. The preamble is used for packet detection and timing acquisition, as well as the estimation of the channel mask used by the energy receiver. It is constructed by first repeating the preamble code $N_{\text {sync }}$ times, and by up-sampling by a factor $L$. The preamble code is a ternary sequence of length 31 . After that, a start frame delimiter (SFD) is added. The SFD is constructed in a similar manner (see [1] for the details). The resulting sequence is then amplitude modulated to produce the preamble signal. The main differences with respect to the data signal are that (1) no timehopping is used and (2) single pulses are transmitted instead of bursts of pulses. Note that for each frequency band, the 802.15.4a standard foresees the use of two different preamble codes.

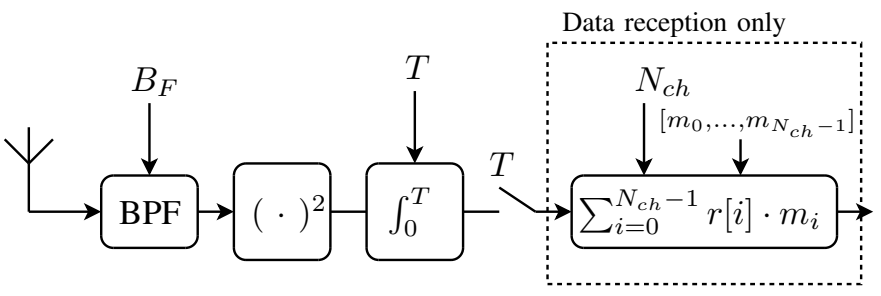

Fig. 1. Architecture of the energy receiver. The antenna is followed by a bandpass filter, a square device, and the integrator. The signal at the output of the integrator is sampled every $T$ seconds. Finally, a scalar product is computed between a block of $N_{c h}$ samples $r[0], \ldots, r\left[N_{c h}-1\right]$ and the channel mask $\left[m_{0}, \ldots, m_{N_{c h}-1}\right]$.

The architecture of the energy-detector receiver is depicted in Figure 1; the antenna is followed by a bandpass filter of bandwidth $B_{F}$, a square device, and the integrator. The signal

\begin{tabular}{c|c|c|c|c|c|c|c}
\hline$T$ & $f_{s}$ & $B_{F}$ & $G$ & $M$ & $N_{\text {ch }}$ & $G_{\text {chest }}$ & $p_{\text {thld }}$ \\
\hline \hline $8 \mathrm{~ns}$ & $125 \mathrm{MHz}$ & $1 \mathrm{GHz}$ & 4 & 5 & 16 & 8 & 0.9999 \\
\hline
\end{tabular}

TABLE I

PARAMETERS OF THE ENERGY-DETECTION RECEIVER

\begin{tabular}{c|c|c|c|c||c|c|c}
\hline \multicolumn{6}{c}{ Mandatory data rate of 0.85 Mbit/s (LPRF mode) } \\
\hline & $N_{c}$ & $T_{c}$ & $N_{c p b}$ & $N_{\text {hop }}$ & $L$ & $N_{\text {sync }}$ & $N_{s f d}$ \\
\hline \hline LPRF & 512 & $2 \mathrm{~ns}$ & 4 & 32 & 64 & 64 & 8 \\
\hline
\end{tabular}

TABLE II

PARAMETERS OF THE PHYSICAL LAYER

$r(t)$ at the output of the integrator is sampled every $T$ seconds to obtain the discrete time signal $r[i]$. Our receiver uses a channel mask to properly set the total integration time per symbol of the energy detection receiver. A channel mask is an estimation of the average power delay profile of the received signal [6]. It also allows for scaling the output of the integrator in order to avoid integrating the received signal when it mostly consists of noise.

The values of the parameters of the receiver are given in Table I. The sampling frequency $f_{s}$ at the output of the receiver is $125 \mathrm{MHz}$ (or a sample period $T$ of $8 \mathrm{~ns}$ ). This value corresponds to the length of a burst in the LPRF mode of the 802.15.4a proposal.

There are three steps for packet detection and timing acquisition: (1) coarse synchronization, (2) fine synchronization, and (3) SFD detection.

During coarse synchronization, we try to locate the starting time of one of the $N_{\text {sync }}$ repetitions of the preamble code. With $L=64$, the timing accuracy of the coarse synchronization phase is $T_{c} L=128 \mathrm{~ns}$. With an integration time of $T=8 \mathrm{~ns}$, the length of a preamble code symbol corresponds to $T_{c} L / T=16$ samples of the discrete time signal $r[i]$. Hence, we first create a correlation template by repeating each of the 31 elements of the preamble code 16 times. We repeat the obtained sequence $G$ times to provide for some processing gain. We then correlate this template with the received signal $r[i]$, and down-sample the result by a factor 16. Finally, we group the output of the correlation by consecutive blocks of 31 elements $\left[\mathcal{R}_{0}[j], \ldots \mathcal{R}_{30}[j]\right]$ and look for the maximum element $R_{\max }[j]=\max \left(\mathcal{R}_{0}[j], \ldots \mathcal{R}_{30}[j]\right)$ within each block. If $R_{\max }[j]$ lies above a certain threshold $\tau_{\text {coarse }}$, we select its position within the $j$ th block as a possible synchronization point. A verification process is started on the subsequent $M$ blocks of 31 elements. If for all the blocks $k=j+1, \ldots, j+M$, we find that $R_{\max }[k]>\tau_{\text {coarse }}$ and that the position of $R_{\max }[k]$ within the $k$ th block is the same as $R_{\max }[j]$, the coarse synchronization succeeds. Assuming additive white Gaussian noise (AWGN) with known variance $\sigma^{2}$ (since it is generated by the circuitry of the receiver), the threshold $\tau_{\text {coarse }}$ can be analytically determined as a function of $\sigma^{2}$. We set $\tau_{\text {coarse }}$ such that the probability that a sequence consisting purely of noise exceeds $\tau_{\text {coarse }}$ is smaller than a certain threshold probability $p_{t h l d}$.

During fine synchronization, we improve the accuracy of the synchronization point to obtain a timing accuracy in the order of the integration time $T$. This is achieved by correlating the received signal $r[i]$ with a finer template and looking for the start of the signal in the vicinity of the coarse synchronization point. The template for the fine synchronization is obtained by 
up-sampling the preamble code by a factor of 16 and repeating the obtained sequence $G$ times.

Between fine synchronization and SFD detection, we estimate the channel mask. It is a sampled and quantized version of the power delay profile of the channel represented as a binary vector $\left[m_{0}, m_{1}, \ldots, m_{N_{c h}-1}\right], m_{i} \in\{0,1\}, \forall i$. The channel mask is used to reduce the amount of noise accumulated by the energy receiver. The estimation is done by averaging $G_{\text {chest }}$ blocks of $N_{c h}$ samples of the received signal. A threshold is then applied to quantize the $N_{c h}$ values to 0 or 1 . This threshold is computed in a similar manner as $\tau_{\text {coarse. }}$ In the same averaging process, we also estimate the received signal level contained in the channel mask.

After the estimation of the channel mask, we begin to look for the SFD sequence. The mandatory 802.15.4a SFD is a sequence of 8 modulated preamble codes scrambled by the so-called SFD code [1]. It is found with a correlation procedure taking into account the noise variance and the estimated received signal level. Note that when searching for the SFD, the channel mask is applied to the received signal.

Due to space restrictions, and because it is not the primary interest of this paper, we cannot explain our synchronization algorithms up to the finest details. However, we do believe that it comes close to what would be used in a real-world implementation of a non-coherent, 802.15.4a compliant receiver. Also, the thresholds ( $\tau_{\text {coarse }}$ and the one for the channel mask quantization) are computed assuming only AWGN and no MUI. Indeed, the receiver has no knowledge of when an interferer might be transmitting or of the statistics of the MUI.

Following the detection of the SFD, we can start decoding the data. For each frame, two scalar products $s_{j}=\sum_{i=0}^{N_{c h}-1} r_{j}[i] \cdot m_{i}, j=0,1$ are computed where $r_{0}[0], \ldots, r_{0}\left[N_{c h}-1\right]$ is the signal where the bit 0 is expected and $r_{1}[0], \ldots, r_{1}\left[N_{c h}-1\right]$ is the signal where the bit 1 is expected. A comparator $1_{\left\{s_{0}>s_{1}\right\}}$ produces a binary output which is fed to the Reed-Solomon decoder.

\section{PERformance Evaluation}

In this section, we evaluate the performance of an IEEE 802.15.4a physical layer that uses the energy detection receiver (described in Section II) in the presence of MUI. We use two different performance metrics; the bit error rate (BER) and the packet error rate (PER).

We perform a packet-based simulation, and we simulate a full IEEE 802.15.4a system with coarse and fine synchronization, estimation of the channel mask, SFD detection, and RS decoding. We denote by $N_{u}$ the number of users that are transmitting packets. For complexity reasons, we cannot simulate the full MAC protocol because this implies to simulate the reception and decoding of every single packet from any user at its destination, as well as the transmission and reception of the acknowledgment packets ${ }^{1}$. Instead, we attempt to decode only the packets from the user of interest. To generate MUI, we consider that each user has a queue with a packet arrival rate $\lambda_{i}, i=0,1, \ldots, N_{u}-1$. When a packet reaches the front of the queue, we draw a backoff time

\footnotetext{
${ }^{1}$ Acknowledgment packets are necessary in order to correctly simulate the backoff algorithm
}

according to the IEEE 802.15.4a procedure with the backoff exponent set to its maximum value. When the backoff expires, the packet is transmitted on the channel. We send 1014 bit per packet: with RS encoding, this corresponds to 1209 symbols. We assume the use of the mandatory frequency band 3, with a center frequency of $4.49 \mathrm{GHz}$. The two possible preamble codes are code 5 and 6 [1].

We simulate the whole physical layer with an accuracy of $100 \mathrm{ps}$ (a simulation sampling frequency of $10 \mathrm{GHz}$ ). This appears to be a good tradeoff between complexity and accuracy. We use the IEEE 802.15.4a channel model 1 [7] (With an RMS delay around $18 \mathrm{~ns}$ ).

In our simulations, we define the signal to noise ratio (SNR) as $\mathrm{SNR}=\frac{E_{p}}{N_{0}}$ where $E_{p}$ is the received energy per pulse (after the convolution of the pulse with the impulse response of the channel), and $N_{0} / 2$ is the variance of a zero mean, Gaussian noise process bandlimited to $B_{F}$. As the simulation sampling frequency is larger than $2 B_{F}$, the Gaussian noise samples are correlated. We use the algorithm in [8] to generate the correlated noise samples.

In Figure 2, we show the PER and BER obtained for a single user (with no MUI). For the BER, there is $1.5 \mathrm{~dB}$ difference with respect to the BER of BPPM on AWGN channels (which is obtained as $Q(\sqrt{S N R})$ assuming a matched filter). We also plot the percentage of missed packets by the synchronization procedure. With a multipath channel and no MUI, we can conclude that the receiver is well balanced between the synchronization procedure and the decoding of the data. Indeed, there are no missed packets above $12 \mathrm{~dB}$ and the BER is below $10^{-5}$ above $14 \mathrm{~dB}$.

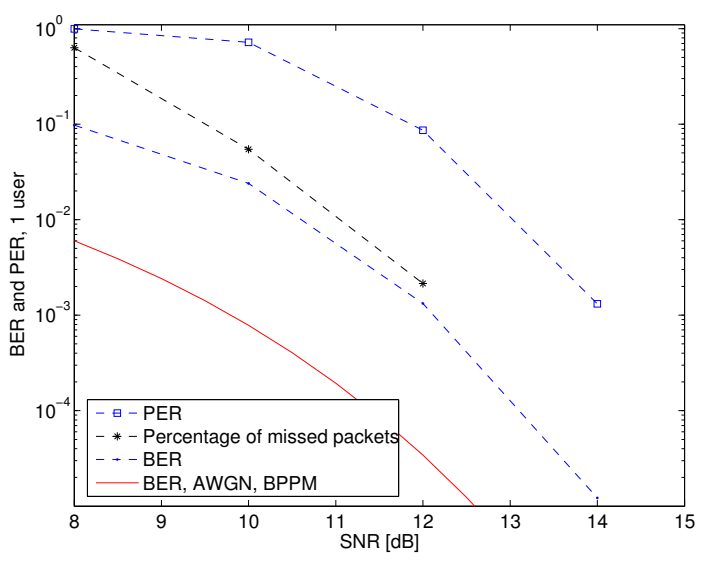

Fig. 2. PER, BER, and percentage of packets missed by the timing acquisition for a single user, on a multipath channel, with no MUI. The plain curve represents the performance of BPPM on an AWGN channel.

For the simulations with MUI, we consider two different scenarios; in scenario A, all the devices use the same preamble code. In scenario $\mathrm{B}$, the user of interest uses preamble code 5 and the other users use preamble code 6 . In both cases, we consider $\lambda_{0}=\lambda_{1}=\lambda$ with either a high traffic case where $\lambda=200$ packet/s, or a low traffic case where $\lambda=10$ packet/s. Note that even in the high traffic case the network is not yet saturated and that $\lambda=200$ packet/s $(\lambda=10$ packet/s, resp.) corresponds to an effective data rate of $241.8 \mathrm{kbit} / \mathrm{s}$ $(12.1 \mathrm{kbit} / \mathrm{s})$. Also, because we are using the maximum backoff 
exponent, our results are independent of the arrival rate of the user of interest $\left(\lambda_{0}=\lambda_{1}=200\right.$ packet/s yields the same results as $\lambda_{0}=10$ packet/s, $\lambda_{1}=200$ packet/s).

In order to understand how robust energy detection is with respect to MUI, we compare the performance obtained by simulations with the performance when considering two very specific capture models. With the "Destructive Collisions" model, a packet is lost whenever there is more than one active transmission at the same time. If there is only one active transmission, single user performance is then assumed. This is the worst case performance. With the "Perfect Capture" model, all active users may compete during packet detection and timing acquisition. Only one of them succeeds (chosen uniformly at random) and this winning user experiences single user performance. This is the ideal performance, which one would obtain if transmissions would be perfectly orthogonal.

In Figure 3 we first show the PER obtained with 2 users with equal received powers, $\lambda=10$ packet/s, in the case of scenario B (different preamble codes). Clearly there is an error-floor with MUI, even in this low traffic case. Further, we look at two more cases with unequal received powers: first, a near-far case, where the received power of the second user is $10 \mathrm{~dB}$ higher; second, a case with 4 users, where the received powers of the three interferers are $3 \mathrm{~dB}$ lower than the received power of the user of interest. We also compare the previous results with those obtained with the "Destructive Collisions" and "Perfect Capture" models. We find that the energy detection receiver operates quite close to the worst case scenario of the "Destructive Collisions" model, and shows only limited capture effect. Its performance is even identical to the "Destructive Collisions" model in the presence of only one strong interferer. Using an energy detector with an 802.15.4a physical layer thus annihilates one of the most appealing benefits of UWB, namely its robustness to MUI and thus, the possibility to allow concurrent transmissions. The conclusions are identical for $\lambda=200$ packet/s.

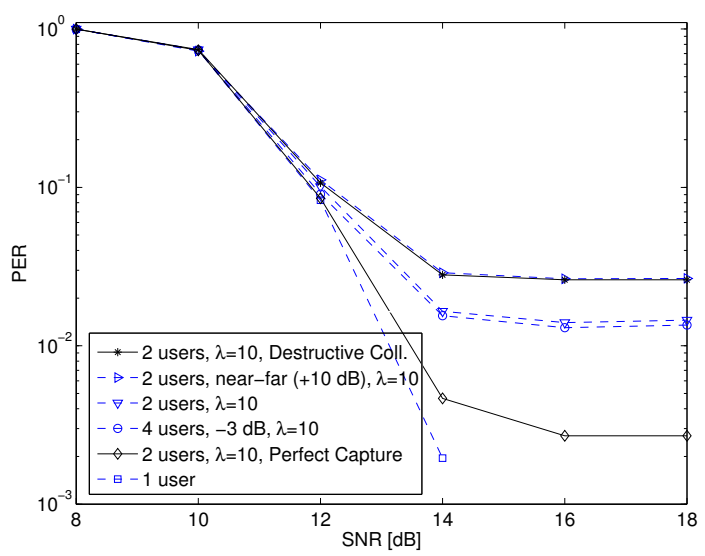

Fig. 3. PER with MUI and different preamble codes (scenario B). Dashed lines from top to bottom: simulation results for: two users in a low traffic case ( $\lambda=10 \mathrm{packet} / \mathrm{s})$, where the received power of the interferers is to $+10 \mathrm{~dB}$ or $0 \mathrm{~dB}$; four users where the received power of the interferers is $-3 \mathrm{~dB}$; no interference. Plain lines: "Destructive Collisions" and "Perfect Capture" models. The performance is close to the "Destructive Collisions" model.

When analyzing packet errors, we observe that they occur for two reasons: (1) a packet is missed during the synchro- nization phase or (2) it is received with more errors than the Reed-Solomon code could correct. In the first case, we can further distinguish two cases:

(1a) We miss the packet because of a missed detection (MD): the receiver is trying to acquire a packet but is not able to acquire timing, or it acquires timing correctly but later misses the SFD.

(1b) We miss the packet because of a false alarm (FA): the receiver is not trying to acquire a packet because it wrongly assumes to be already successfully synchronized.

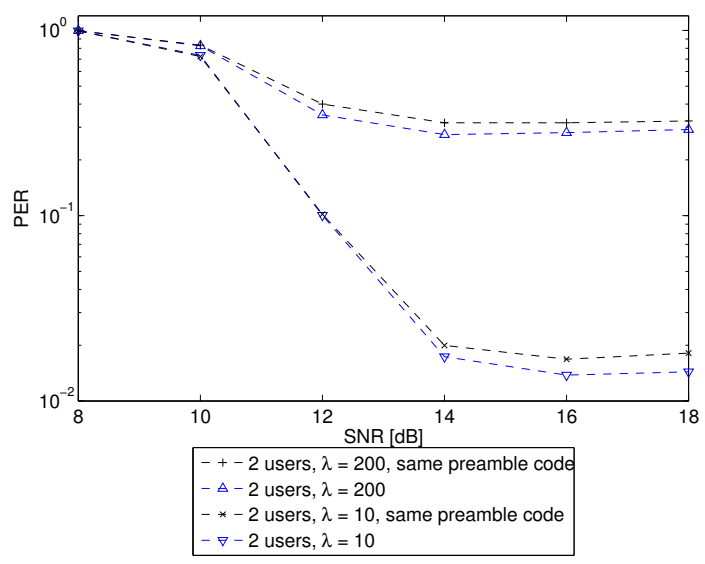

Fig. 4. Comparison of the PER when two users use the same preamble code or different preamble codes. The two users have equal power at the receiver. We show a high $(\lambda=200$ packet/s $)$ and a low traffic case $(\lambda=10$ packet/s $)$. There is a negligible difference whether we use the same preamble code or a different preamble code.

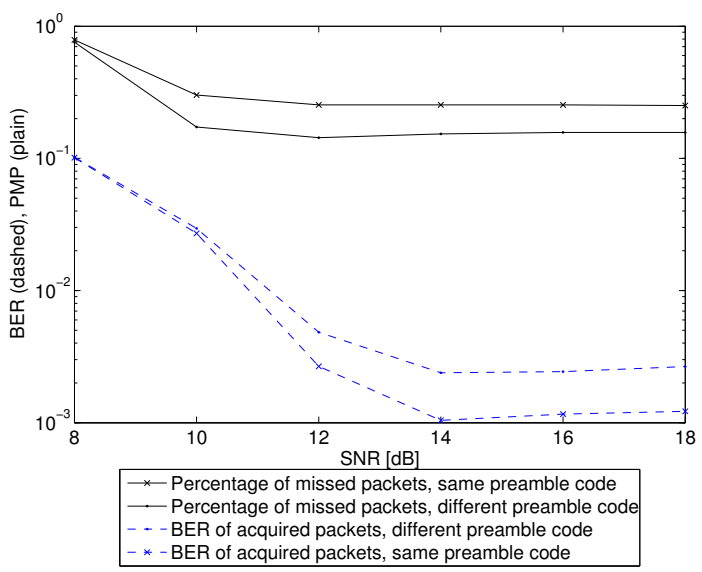

Fig. 5. Percentage of missed packets (PMP, plain lines) and BER (dashed lines) when two users use the same or a different preamble code. We consider the high traffic case $(\lambda=200$ packets/s). If different codes are being used, there are less packets missed. However, the packets additionally acquired, have generally more interference, which translates into a higher BER.

Now, if both the interferer and the user of interest use the same preamble code (scenario A), the receiver will miss lots of packets due to FAs. Hence, we expect a much worse performance compared to the case where they use different codes (scenario B). A comparison of these two scenarios is shown in Figure 4 where it can be seen that the difference is surprisingly small. Looking at the percentage of packets missed during the synchronization phase (see Figure 5) confirms that the receiver misses more packets in scenario A than 


\begin{tabular}{l|c|c}
\hline & Same Code & Different Code \\
\hline \hline Percentage of missed packets & $25.3 \%$ & $15.7 \%$ \\
\hline $\begin{array}{l}\text { Out of which missed due to } \\
\text { false alarm (FA) }\end{array}$ & $98.0 \%$ & $52.1 \%$ \\
\hline $\begin{array}{l}\text { Out of which FA due to } \\
\text { wrong timing acquisition }\end{array}$ & $99.0 \%$ & $99.1 \%$ \\
\hline
\end{tabular}

TABLE III

PERCENTAGE OF MISSED PACKETS AND CLASSIFICATION OF THE REASON THEY WERE MISSED. NUMBERS SHOWN ARE FOR PACKET ARRIVAL RATE OF $\lambda=200$ packet/s AND AT AN SNR OF $18 \mathrm{~dB}$

in scenario B. We can further see in Table III that of the missed packets in scenario A, $98 \%$ are effectively missed because of a FA. However, if we consider the BER of the packets that are correctly acquired (see Figure 5), we notice that the acquired packets generally have more errors in scenario B than scenario A. We conclude that the packets additionally acquired by the receiver in scenario B are packets with a lot of interference. Therefore, a lower rate of missed packets does not translate to a huge performance improvement in terms of PER.

Another observation that may come as a surprise is the fact that even in scenario B, more than $50 \%$ of the missed packets are missed because of a FA (see Table III). Let us classify FAs into two categories:

(1) The receiver acquires timing correctly but wrongly declares detection of the SFD. This can happen if noise or the signal of the interferer make the receiver exceed the SFD detection threshold even though the SFD of the user of interest is not present.

(2) Noise and interference lead the receiver to acquire a wrong timing and wrongly declare detection of an SFD.

Analyzing FAs in scenario B, we observe that more than $90 \%$ of them fall into category 2 . Hence, it seems that even if the receiver and the interferer use a different preamble code, the receiver still synchronizes with the interferer often. A reason for this behavior becomes apparent if we look at the correlation between preamble codes. In Figure 6(a), we first show the correlation of preamble code 5 (the correlation template) with a periodic repetition of itself (representing the sequence of the user of interest) and secondly with a periodic repetition of preamble code 6 (representing the sequence of an interferer) $)^{2}$. We observe that the first correlation has a peak only when there is a perfect alignment between the sequence and the template. On the other hand, there are 10 out of the 31 possible shifts that lead to a peak in the correlation of the interferer sequence and the template. Even though these peaks are lower when the sequence does not match the template, they might (depending on the signal level of the interferer and the noise) still exceed the detection threshold of the coarse synchronization phase and consequently lead to FAs. To verify this assumption, we classify wrong timing acquisitions in our simulations according to the offset (in number of code symbols) with respect to the closest packet (in time) of an interferer. The result for $\lambda=200$ packet/s at an SNR of 18 $\mathrm{dB}$ is shown in Figure 6(b); the offsets with the largest number of packets correspond exactly to the offsets producing peaks in the correlation.

\footnotetext{
${ }^{2}$ Note that in the case of an energy-detector receiver, the ternary preamble code sequence is transformed into a binary one because of the squaring operation.
}

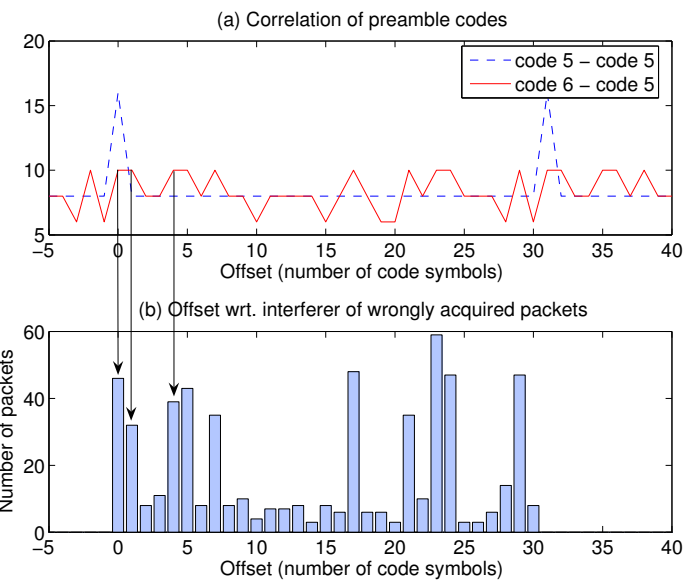

Fig. 6. (a, top) Correlation of preamble code 5 with a periodic repetition of itself and with a periodic repetition of preamble code 6 . (b, bottom) Classification of wrong timing acquisitions according to the offset with respect to the closest packet of an interferer. Correspondence of peaks in (a) and (b) suggests that wrong timing acquisitions are due to the correlation properties of the preamble codes.

In Figure 7, we compare the PER shown in Figure 3, with a PER obtained with a perfect synchronization and channel mask estimation algorithm; an oracle returns the exact beginning of the packet of the user of interest. Hence, there are no FAs or MDs. Besides, the estimation of the channel mask assumes perfect synchronization and no MUI. Thus, Figure 7 allows for assessing whether the performance degradation is solely due to the synchronization phase or whether MUI also significantly affects the data decoding phase. Even in the case of perfect synchronization, there is a clear error floor. MUI during synchronization and MUI during data decoding are equally responsible for the performance degradation.

It can also be observed in Figure 7 that the error-floor of the BER shows an increasing trend after $14 \mathrm{~dB}$. Table IV shows that the average number of ones in the channel mask increases proportionally with the SNR. A large number of ones in the channel mask implies a higher likelihood of suffering from MUI as we integrate a larger amount of the received signal.

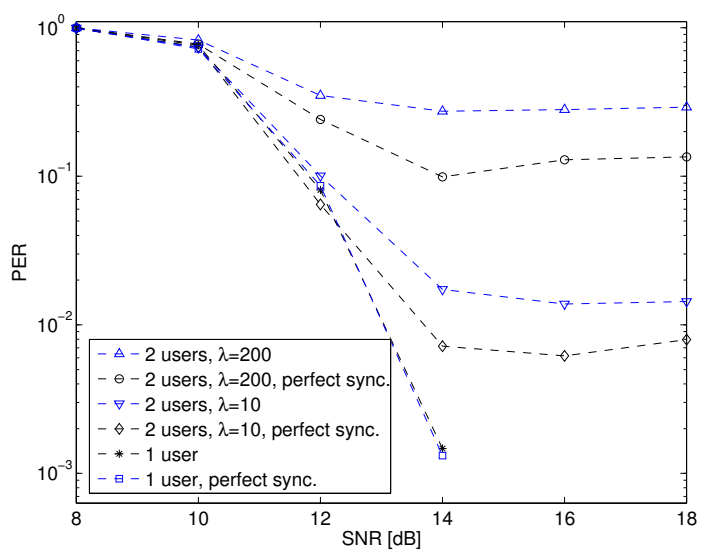

Fig. 7. Comparison of the PER between our implementation of the synchronization and channel mask estimation algorithm and a perfect synchronization and channel mask estimation. The perfect channel mask estimation assumes no MUI.

The preamble and payload of an 802.15.4a packet have a different format. Hence, it is interesting to assess whether packet errors are caused by an interfering preamble or an 


\begin{tabular}{l|c|c|c|c|c|c}
\hline \multicolumn{1}{c}{ Avg. number of ones in channel mask (perfect synchronization) } \\
\hline Correct packets & 1.00 & 2.17 & 3.30 & 4.28 & 5.09 & 5.97 \\
Erroneous packets & 1.61 & 2.39 & 3.15 & 4.52 & 5.58 & 6.08 \\
\hline SNR [dB] & 8 & 10 & 12 & 14 & 16 & 18 \\
\hline
\end{tabular}

TABLE IV

AVERAGE NUMBER OF ONES IN CHANNEL MASK (PERFECT SYNCHRONIZATION, 2 USERS WITH $\lambda=200$ PACKET/S)

interfering payload. The following analysis is done with the interferer having an equal received power but using a different preamble code than the user of interest. The SNR is $18 \mathrm{~dB}$ and $\lambda=200$ packet/s (PER shown in Figure 3). Our conclusions are similar for other cases presented in this paper. Looking first at packets that are correctly acquired but incorrectly decoded, $91.3 \%$ of these packets have an overlap of more than $30 \%$ with an interfering payload. Moreover, $46.1 \%$ of these packets have no overlap at all with an interfering preamble. We conclude that errors in this case are mostly due to an interfering payload.

We then look at packets that are missed during the synchronization phase. Table III, Figure 6 and the corresponding analysis already demonstrated that false alarms mostly happen because the receiver synchronizes with an interfering preamble. Missed detections (MD) occur if (1) the receiver is not able to acquire timing, or (2) it acquires timing but later misses the SFD. In our simulations, $92.6 \%$ of MDs are of type 2, the remaining $7.4 \%$ are of type 1 . For both types, on average $80 \%$ of the preamble overlaps with an interfering payload. We conclude that MDs are also mostly due to interference with a payload.

The above results suggest that most of the packet errors are due to an interfering payload. This also makes sense from the perspective that a burst in the payload contains four times more energy than a pulse of the preamble. Furthermore, the payload is much longer than the preamble, making a collision with a payload more likely.

\section{RELATED WORK}

The robustness of an energy detection receiver with an IR-UWB physical layer has been demonstrated in [3], [9]. Both [10] and [3] considered packet detection and timing acquisition. They exposed the excellent trade-off between complexity and performance of an energy detection receiver for IR-UWB.

Many papers recognize that it is necessary to adapt integration window of the energy detection receiver to the characteristics of the received signal. For code acquisition, [11] studied the use of a non-coherent Rake receiver; how many paths should be combined depending on the channel characteristics, how they should be combined, and the effect on the probability of false acquisition and missed detection. There are several proposals of energy detection receivers where the integration time is adapted [12], [13], [14], [15], [16]. There are more sophisticated approaches [6], [17], [18], where the authors take advantage of partial channel state information in general for designing the receiver (and not only adapting the integration time). The work in [6] is very similar to the concept of a channel mask that we use in this article.

Note that none of the previous work considers MUI or the performance evaluation of a complete system, with both synchronization and data reception.

\section{CONCLUSiON}

We have evaluated the effect of MUI on an IEEE 802.15.4a physical layer that uses an energy detection receiver. Our results show that an energy detection receiver is a good and well-balanced solution if no MUI is present. However, already in low traffic cases, the performance is severely degraded in presence of MUI. One of the most appealing benefits of UWB, namely its robustness to MUI and thus the possibility to allow parallel transmissions is completely annihilated by the energy detection receiver. Both timing acquisition and data decoding are affected. For future work, it might be interesting to take into account the CCA mode 5 and 6 [1]. We will also compare our results with those obtained when using a realistic coherent receiver. Finally, we will explore the possibilities to mitigate the effect of MUI, even with a low-complexity receiver.

\section{ACKNOWLEDGMENTS}

We would like to thank Armin Wellig, from STMicroelectronics, for all the discussions and comments on this paper.

\section{REFERENCES}

[1] "IEEE P802.15.4a/D5 (amendment of IEEE std 802.15.4), part 15.4: Wireless medium access control (MAC) and physical layer (PHY) specifications for low-rate wireless personal area networks (LRWPANs)," September 2006.

[2] M. Z. Win and R. A. Scholtz, "Impulse radio: how it works," IEEE Communications Letters, vol. 2, no. 2, pp. 36-38, 1998.

[3] S. Dubouloz, A. Rabbachin, S. de Rivaz, B. Denis, and L. Ouvry, "Performance analysis of low complexity solutions for UWB low data rate impulse radio," in IEEE ISCAS 06, May 2006.

[4] V. Lottici, A. D'Andrea, and U. Mengali, "Channel estimation for ultra-wideband communications," IEEE Journal on Selected Areas in Communications, vol. 20, no. 9, pp. 1638-1645, 2002.

[5] "UWB research at EPFL-IC," http://icawww1.epfl.ch/uwb/, 2007.

[6] Z. Tian and B. Sadler, "Weighted energy detection of ultra-wideband signals," in IEEE SPAWC 05, June 2005, pp. $10168-1072$.

[7] "IEEE 802.15.4a channel model - final report, document 04/662r0," http://www.ieee802.org/15/pub/TG4a.html, November 2004.

[8] N. C. Beaulieu and C. C. Tan, "An FFT method for generating bandlimited gaussian noise variates," in IEEE GLOBECOM 97, vol. 2, 1997.

[9] F. S. Lee and A. P. Chandrakasan, "A 2.5nj/b 0.65v 3-to-5ghz subbanded UWB receiver in 90nm CMOS," in ISSCC 07, February 2007.

[10] A. Rabbachin and I. Oppermann, "Synchronization analysis for UWB systems with a low-complexity energy collection receiver," in IEEE joint UWBST \& IWUWBS 04, 2004, pp. 288-292.

[11] M. Villanti, M. Sabattini, G. Maggio, and L. Milstein, "Non-coherent code acquisition for UWB systems in dense multipath fading channels," in IEEE Spring VTC 05, vol. 3, June 2005, pp. 2018 - 2022.

[12] M. Weisenhorn and W. Hirt, "Robust noncoherent receiver exploiting UWB channel properties," in IEEE joint UWBST \& IWUWBS, 2004.

[13] S. Paquelet and L. M. Aubert, "An energy adaptive demodulation for high data rates with impulse radio," 2004, pp. 323-326.

[14] M. E. Sahin, I. Guvenc, and H. Arslan, "Optimization of energy detector receivers for UWB systems," in IEEE Spring VTC 05, vol. 2, 2005.

[15] M. Nemati, U. Mitra, and R. Scholtz, "Optimum integration time for UWB transmitted reference and energy detector receivers," in IEEE MILCOM 06, October 2006, pp. 1-7.

[16] X. Peng, F. Chin, S. H. Wong, K. Y. Sam, and L. Zhongding, "A rake combining scheme for an energy detection based noncoherent OOK receiver in UWB impulse radio systems," in IEEE ICUWB 06, September 2006, pp. 73-78.

[17] E. Arias-De-Reyna, A. A. D'Amico, and U. Mengali, "UWB energy detection receivers with partial channel knowledge," in IEEE ICC 06 , vol. 10, 2006, pp. 4688-4693.

[18] T. Zasowski, F. Troesch, and A. Wittneben, "Partial channel state information and intersymbol interference in low complexity UWB PPM detection," in IEEE ICUWB 06, September 2006, pp. $369-374$. 